\title{
Associations between attitudes toward cosmetic surgery, celebrity worship, and body image among South Korean and US female college students
}

\author{
Jaehee Jung $^{1 *}$ and Choon Sup Hwang ${ }^{2}$
}

\section{${ }^{*}$ Correspondence:}

jajung@udel.edu

${ }^{1}$ University of Delaware,

Newark, USA

Full list of author information

is available at the end of the article

\begin{abstract}
Very little information is known regarding the level of psychological attachment to celebrities needed to influence one's desire to engage in cosmetic surgery in consumers. More importantly, no previous research examined this in relation to attitudes toward cosmetic surgery and level of body satisfaction in cross-cultural contexts. The present study aimed to examine associations between attitudes toward cosmetic surgery, celebrity worship, and body image among South Korean and US female college students. A total of 370 female undergraduates were recruited for survey from a central university in Seoul, South Korea $(n=196)$ and a mid-Atlantic university in the US $(n=174)$. The South Korean participants showed greater acceptance toward cosmetic surgery than did the US participants, whereas the US participants showed more positive attitudes toward their favorite celebrities than did the South Korean participants. For the US participants, the level of body dissatisfaction was negatively associated with acceptance for cosmetic surgery while attitudes toward favorite celebrities was positively associated with acceptance for cosmetic surgery. Findings suggest that cultural contexts affect factors influencing the likelihood of having cosmetic surgery.
\end{abstract}

\section{Introduction}

Cosmetic and plastic surgery procedures, such as liposuction, rhinoplasty (nose reshaping), and breast augmentation, and minimally invasive procedures, such as Botox injections and chemical peels, continue to increase in popularity in the West (American Society of Plastic Surgeons 2014). According to the American Society of Plastic Surgeons (ASPS), the world's largest organization of board-certified plastic surgeons, 15.1 million cosmetic surgery procedures, excluding reconstructive surgery procedures, were performed in the United States in 2013 (American Society of Plastic Surgeons 2014). The American Society for Aesthetic Plastic Surgery (2015) reports that Americans spent more than 12 billion dollars on surgical and nonsurgical (i.e., minimally-invasive) procedures in 2014. The top five cosmetic surgical procedures were liposuction, breast augmentation, eyelid surgery, tummy tuck and rhinoplasty. Increased popularity of cosmetic surgery has been attributed to a number of factors such as the increased importance of physical appearance in contemporary societies, the lower cost of procedures, and 
increased public awareness of cosmetic surgery through media (Edmonds 2007; Sarwer et al. 2007; Sarwer and Magee 2006). In addition, new products and advances in technology seem to boost steady growth in the US cosmetic surgery rate (American Society of Plastic Surgeons 2014). With the increasing rates of cosmetic surgery, some researchers have sought to understand psychological factors that influence some individuals but not others to consider having cosmetic surgery. Past research has revealed that there are reliable associations between the consideration of cosmetic surgery and lower self-ratings of physical attractiveness and negative body image (Brown et al. 2007; Crerand et al. 2006; Swami et al. 2009a). Similar to the finding that media are known to negatively influence body image, greater media exposure was associated with greater willingness to undergo cosmetic surgery (Swami et al. 2008a).

There has been an explosion in the number of cosmetic procedures taking place in non-Western societies (ISAPS Global Statistics 2014), and yet most of the studies on cosmetic surgery have relied on Western samples and there continues to be a lack of research on acceptance of cosmetic surgery in non-Western populations. Also, research within these populations has also been constrained due to scarce information about cultural differences on attitudes toward cosmetic surgery and their relationships with body image. Among the sociocultural factors influencing body image, media influences on body image in general are well-documented in the literature (e.g., Durkin et al. 2007; Grabe et al. 2008). However, the exploration of how idolization of specific media personnel (e.g., celebrities) can lead to extreme behaviors is under-researched. One's desire to alter own physical characteristics to make them similar to those of celebrities is considered an extreme form of 'celebrity worship', or the idolization of celebrities as role models (Giles 2002). Very little information is known regarding the level of psychological attachment to celebrities needed to influence one's desire to engage in cosmetic surgery in consumers. Thus, the examination of such relationship is necessary to better understand factors influencing the epidemic proportions of cosmetic surgery practice in contemporary society.

Among non-Western societies, South Korea has one of the highest rates of cosmetic surgery in the world (White 2005), which is believed to be the manifestation of negative body image. The cosmetic surgery epidemic in South Korea is particularly dramatic because of its sharp contrast with the Confucian philosophy that has dominated Korean social, political, and family life for over 500 years (Bell and Chaibong 2003). Confucian tradition seems to have discouraged body modification procedures since the human body was considered sacred as a symbol of high respect for the ancestors (Bell and Chaibong 2003). However, the Westernization of Korea in the 1980s has resulted in contemporary Korean society adopting more Western appearance standards (Shin and Rutkowski 2003). According to the most recent statistics available at the International Society of American society of plastic surgeons (2014), South Korea is ranked 4th in the world by the total number of surgical and non-surgical procedures following the US, Brazil, and Japan. Despite the evidence that rates of cosmetic surgery in South Korea have increased sharply in the past decade, much of the available research continues to document rates of specific procedures (e.g., McCurdy and Lam 2005) rather than to examine individual or socio-cultural factors influencing cosmetic surgery procedures. According to the empirical evidence, South Korean women and girls have greater body 
dissatisfaction than women and girls in the United States (e.g., Forbes and Jung 2008; Jung et al. 2009). A study by Jung and Forbes (2006) suggests that women in cultures such as South Korea, where increased opportunities for women produced a marked shift toward gender equality, are likely to experience increased pressures to conform to unrealistic appearance standards. With evidence of greater body image issues in South Korea compared to the US, it would be interesting to see whether a greater desire to alter one's appearance through cosmetic procedures also exists in South Korea compared to the US. More importantly, no previous research has examined associations between cosmetic surgery and the extent of celebrity worship and body image across samples in South Korea and the US.

The present study, therefore, aims to examine associations between attitudes toward cosmetic surgery, celebrity worship, and body image among South Korean and US female college students. This study is intended to shed light on this under-researched topic in the literature since there is no research that has examined all three of these variables in these two countries. While cosmetic surgery is widely popular in both South Korea and the US, the two countries have contrasting cultural backgrounds due to the values and beliefs associated with being an Eastern and a Western culture, respectively. Therefore, the outcome of this study should be valuable for understanding cultural attitudes on selected variables considering the risks involved with surgical procedures. With a lack of knowledge on this topic, the outcome of this study will add to the literature on cosmetic surgery and celebrity worship for cross-cultural applications.

\section{Literature review}

With advances in medical technologies and higher disposable incomes, there has been an increasing demand for various procedures across many different demographics. The way in which cosmetic surgery is portrayed in the mass media and entertainment industries seems to have reduced negative social stigmas associated with cosmetic surgery (Swami et al. 2008a). For example, various television programs, including reality shows focusing on media personnel (e.g., Keeping up with the Kardashians) and housewives in metropolitan cities of the US (e.g., The Real Housewives of Los Angeles), and magazine and Internet advertisements frequently feature procedures that are used to enhance one's appearance to have a more youthful and culturally idealized appearance. According to the American Society for Aesthetic Plastic Surgery's statistics (2014), buttock augmentation procedures increased in number by $86 \%$ in 2014. This indicates the influence of reality TV stars (e.g., Kim Kardashian) on cosmetic surgery procedures.

The fashion industry is considered one of the most important industries associated with the social phenomenon of cosmetic surgery, despite emerging diversity among models in the contemporary media. Models used for advertising fashion products still predominantly endorse the cultural standards of ideal beauty (Sypeck et al. 2004) that are deviant from the appearance norms of the majority. For most individuals, the ideal beauty standards propagated through media images are not obtainable by natural means. The mainstream media are pervasive with images of a slim figure having full-breasts or a small waist with full buttocks, known as the curvaceously thin woman (Harrison 2003). Since achieving this ideal figure would be difficult or impossible, some women 
may attempt to improve their physical appearance (Delinksy 2005) through cosmetic procedures.

\section{Previous findings on cosmetic surgery}

Although studies exist on cosmetic surgery in regards to reasons for seeking cosmetic surgery and possible psychological results of such surgery, this literature was mostly motivated by the medical community for the need to assess the psychological suitability of patients for various procedures (Cook et al. 2006). Contrastingly, research on factors influencing the future likelihood of having cosmetic surgery is still sparse, and there is a need to overcome this deficiency in the literature because of the general interest in cosmetic surgery among women and men (Frederick et al. 2007; Sarwer et al. 2005).

According to previous findings focused on factors influencing the likelihood of having cosmetic surgery, gender seems to be an influencing factor since women were more likely to consider most cosmetic procedures than men (Brown et al. 2007; Swami 2008a). Brown et al. (2007) found that lower self-ratings of physical attractiveness predicted a higher likelihood of having cosmetic surgery. This supports the notion that failure to attain cultural body ideals may lead to greater body dissatisfaction and possibly to the consideration of cosmetic surgery. In addition, previous experience of having had cosmetic surgery was a significant predictor for future likelihood of augmentative procedures (Brown et al. 2007). Media exposure (viewing advertisements, television programs, or reading articles about cosmetic surgery), as a factor affecting the likelihood of having cosmetic surgery, has resulted in somewhat inconsistent outcomes. While findings from the Brown et al.s (2007) study revealed no effect of media exposure on the likelihood of having cosmetic surgery, findings from Swami et al. (2008a) indicated an important role for media exposure in mediating the effects of participants' sex and previous personal experience of cosmetic surgery. This suggests that media exposure itself may not be directly associated with consumer attitudes toward cosmetic surgery such as willingness to have cosmetic surgery.

\section{South Korea}

Recent empirical evidence shows that body dissatisfaction and disordered eating are not uncommon in East Asian societies, particularly in South Korea (e.g., Jung and Forbes 2006; Jung and Forbes 2006). In a comparison of samples of Korean and US college women, Jung and Forbes (2006) found that the Korean sample scored higher than the US sample in multidimensional measures of body dissatisfaction and disordered eating. In fact, their findings were consistent with other evidence of a high level of body and weight dissatisfaction among adolescents and young women in Korea (Kim and Kim 2003; Rye et al. 2003). Due to the rapid modernization of the past few decades, women in Korea have gained increasing social opportunities such as greater economic and political power. This has transformed Korea from a traditional patriarchal society, where women's roles were primarily bound to the household, to a modern industrialized society, where women's rights and roles in society are important for quality of life.

With an influx of Western media images and practices since the 1990s (Shim 2006), South Korean women seem to experience increased pressures to conform to unrealistic appearance standards that emulate Western body ideals. These pressures, in turn, seem 
to have resulted in increased body dissatisfaction and increased symptoms of eating disorders. In addition, the popularity of the Korean popular culture (Lee 2011), also known as K-pop, featuring flawless and youthful appearance of Korean celebrities (e.g., Girls' Generation) in media outlets, such as TV and music videos, is likely to negatively affect South Korean women's body image and encourage modification of physical characteristics to similar features of K-pop stars. In order to cope with such body image issues, many young Korean women and men are looking to improve their appearances through various surgical procedures such as rhinoplasty, eyelid surgery and breast augmentation, and nonsurgical, minimally-invasive procedures such as laser hair removal and Botox injections (ISAPS Global Statistics 2014). In 2009, one of every five women in Seoul between the ages of 19 and 49 had undergone cosmetic surgery, and a 2010 government survey shows 31.5 percent of residents 15 or older were willing to undergo surgery to improve their looks (Choe 2011). Celebrities are influencing this trend as they attempt to remedy physical imperfections with cosmetic procedures and this seems to be encouraging decisions made by the general public (Choe 2011).

\section{Theoretical framework on celebrity worship and body image}

One particular aspect of media influence that calls for attention, due to its underresearched association with cosmetic surgery, is celebrity worship. The most prominent theoretical account regarding celebrity worship was proposed by McCutcheon et al. (2002), who postulated an "absorption-addiction" model that explains three increasingly extreme sets of cognitions associated with para-social relationships. Celebrity worship is described as a para-social relationship because it is one-sided relationship in which an individual knows the other, but the other does not know the individual (Maltby and Day 2011). In the first instance, 'Entertainment-social' celebrity worship reflects the social aspects of para-social attachment and is driven by an attraction to a favorite celebrity because of their perceived ability to entertain. For some individuals, this attachment may lead to psychological absorption (intensive and compulsive feelings) with a celebrity, or what has been termed 'Intense-personal' celebrity worship attitudes. In extreme cases, this absorption may become addictive, leading to 'Borderline-pathological' celebrity worship attitudes and behaviors that serve to maintain an individual's satisfaction with the para-social attachment (Giles and Maltby 2004; McCutcheon et al. 2002).

Because celebrities in the media pursue youthful appearances, idolization of celebrities would negatively influence one's body image and can lead to a desire for emulating appearance of idolized celebrities. It has been suggested that a desire to look like idealized media icons may result in negative body image when those bodily ideals are not attained (Greenwood 2009). Considering its importance, celebrity worship has been conceptualized as a part of identity-development (e.g., Giles and Maltby 2004). Possible effects on corporeal experiences, including body image disturbance, have been shown through an empirical body of work revealing an association between celebrity worship and poor self-image, symptoms of eating disorders (Greenwood 2009; Shorter et al. 2008), and acceptance of cosmetic surgery (Swami et al. 2009b). Limited studies, nearly all in single country applications, explored the relationship between celebrities and elective cosmetic surgery (e.g., Maltby and Day 2011; Swami et al. 2009b), and despite the reasonable argument, there is still a lack of support for the notion that celebrity worship 
is a strong predictor for positive attitudes toward cosmetic surgery. However, one aspect of celebrity worship has emerged as the strongest predictor, which is the intense-personal celebrity worship. For example, Maltby and Day (2011) found that intense-personal worship of a celebrity whose body shape was admired by the participants predicted the incidence of elective cosmetic surgery.

\section{The present study}

The present study aimed to examine associations between attitudes toward cosmetic surgery, celebrity worship, and body image among South Korean and US female college students. This study exclusively focused on women, as gender was identified as a factor influencing likelihood of having cosmetic surgery procedures and resulted in more women than men as subjects of cosmetic surgery (Brown et al. 2007). While cosmetic procedures are also growing in popularity among men, women still account for 90.5 percent of total procedures in 2015 (Dent 2016). Despite that it is not a representative sample of women, college-aged women were selected for convenience and their great interest in celebrities. Abraham and Zuckerman (2011) reported that cosmetic surgery is becoming widely popular among adolescents and young adults, so the selected age group is believed to be appropriate for this study. This study focused on assessing possible relationships between celebrity worship and attitudes toward cosmetic surgery in the cross-cultural context, along with other variables that have shown positive associations with cosmetic surgery. Other variables included measures of body image and sociocultural influences on body image, specifically media influences on the internalization of body ideals.

Based on previous work on selected variables for this study, the following hypotheses were formulated:

H1: The South Korean participants will show greater body dissatisfaction than the US participants.

H2: The South Korean participants will show greater acceptance of cosmetic surgery than than the US participants.

H3: The South Korean participants will show greater celebrity worship than the US participants.

H4: There will be associations between body image and celerity worship and cosmetic surgery across the two countries.

H4-a: The more negative body image participants show the greater likelihood they will engage in cosmetic surgery.

H4-b: The more celebrity worship participants show the greater likelihood they will engage in cosmetic surgery.

H4-c: The more media influences are on participants' body image the greater likelihood they will engage in cosmetic surgery.

\section{Methods}

\section{Participants and procedure}

A total of 370 female undergraduates were recruited for a survey from a South Korean university $(n=196)$ and a university in the mid-Atlantic region of the US $(n=174)$ upon obtaining the IRB approval from the respective universities. All participants were 
volunteers who were recruited from undergraduate courses throughout the university campus. An independent translator, unaffiliated with the study, translated the initial questionnaire constructed in English into the Korean version, and this version was then back-translated into English by the second author and an independent translator unaffiliated with the study. Minor differences that emerged during this process were resolved between translators and by a bilingual scholar in the US.

While all Korean participants were of Korean ethnicity, the US participants self-identified their ethnicity as White (86.8 \%), Asian (6.3\%) and other (6.9\%). The age of the Korean participants $(M=21.94, S D=1.77)$ was greater than the age of the US participants $(M=20.33, S D=2.43), F(1,368)=53.90, p<.001$. The self-reported weight of the Korean sample [ $(M=51.78, S D=6.46(\mathrm{~kg})]$ was less than that of the US sample $[M=59.79, S D=9.59(\mathrm{~kg})], F(1,368)=90.47, p<.001$. The US participants $[(M=165.60, S D=7.32(\mathrm{~cm})]$ showed greater self-reported height than that of the Korean participants $[(M=162.68, S D=4.69(\mathrm{~cm})], \mathrm{F}(1,368)=21.32, p<.001$. The participants' body mass index [BMI; weight $(\mathrm{kg}) /$ height $\left.^{2}(\mathrm{~m})\right]$, as a proxy for body fat, revealed that the Korean participants $(M=19.56, S D=2.31)$ had less body fat than the US participants $(M=21.74, S D=3.12), F(1,368)=59.19, p<.001$. Sample characteristics based on one-way analysis of variance (ANOVA) are provided in Table 1.

\section{Instruments}

In addition to demographic details, consisting of sex, age, ethnicity, and self-reported height and weight, the participants were asked to complete questionnaires assessing participants' attitudes toward celebrities, acceptance of cosmetic surgery, measures of body satisfaction, and sociocultural influences on body image. Attitudes toward celebrities were measured using the celebrity attitude scale (CAS; McCutcheon et al. 2002) and acceptance of cosmetic surgery was measured using the Acceptance of Cosmetic Surgery Scale (ACSS; Henderson-King and Henderson-King 2005). Measures of body satisfaction included the photographic figure rating scale (PFRS; Swami and ChamorroPremuzic 2008) and the body appreciation scale (Avalos et al. 2005). Sociocultural influences on body image were assessed using the sociocultural attitudes towards appearance questionnaire-3 (SATAQ-3; Thompson et al. 2004). Details on each of the measures being employed are as follows:

Table 1 Sample characteristics

\begin{tabular}{|c|c|c|c|c|c|c|}
\hline \multirow[t]{2}{*}{ Measures } & \multicolumn{2}{|c|}{ Korea $(n=196)$} & \multicolumn{2}{|c|}{ US ( $n=174)$} & \multirow[t]{2}{*}{$F(1,368)$} & \multirow[t]{2}{*}{ Partial eta $^{2}$} \\
\hline & $M$ & $S D$ & $M$ & $S D$ & & \\
\hline Age & 21.94 & 1.77 & 20.33 & 2.43 & $53.90^{* * *}$ & .128 \\
\hline Weight (kg) & 51.78 & 6.46 & 59.79 & 9.59 & $90.47^{* * *}$ & .197 \\
\hline Height (cm) & 162.68 & 4.69 & 165.60 & 7.32 & $21.32^{* * *}$ & .055 \\
\hline $\mathrm{BMI}\left(\mathrm{kg} / \mathrm{m}^{2}\right)$ & 19.56 & 2.31 & 21.74 & 3.12 & $59.19^{* * *}$ & .139 \\
\hline
\end{tabular}


The celebrity attitude scale (CAS; McCutcheon et al. 2002). The CAS is a 34-item measure in which respondents are asked to indicate their attitude toward their favorite celebrity on a 5 -point scale $(1=$ Strongly disagree; $5=$ Strongly agree $)$. During an oral instruction about the questionnaires, participants were asked to select a same-sex living celebrity whose body/figure they liked and admired, similar to previous research (Maltby and Day 2011). Among Western samples, the scale has been found to have a three-factor structure comprising Entertainment-social (e.g., "Keeping up with news about my favorite celebrity is an entertaining pastime"), Intense-personal (e.g., "To know my favorite celebrity is to love him/her"), and Borderline-pathological (e.g., "I have frequent thoughts about my favorite celebrity, even when I don't want to"). Because previous studies with non-Western samples (e.g., Swami et al. 2012b) showed that the three components of the CAS were highly correlated with one another, this study used an overall CAS score for data analysis. Cronbach's coefficient alphas for the overall CAS were Korea $=.94$ and US $=.93$.

The Acceptance of Cosmetic Surgery Scale (Henderson-King and Henderson-King 2005). The 15-item ACSS is the most widely used scale for the measurement of attitudes toward cosmetic surgery. Among Western samples, it has been found to have three subscales, namely Intrapersonal (5 items measuring attitudes related to the self-oriented benefits of cosmetic surgery; e.g., 'Cosmetic surgery is a good thing because it can help people feel better about themselves'), Social (5 items measuring social motivations for having cosmetic surgery; e.g., 'I would seriously consider having cosmetic surgery if I thought my partner would find me more attractive'), and Consider (5 items measuring the likelihood that a participant would consider having cosmetic surgery; e.g., 'If I could have a surgical procedure done for free, I would consider trying cosmetic surgery') (Henderson-King and Henderson-King 2005; Swami et al. 2012a). Items in the ACSS are rated on a 7 -point scale $(1=$ Strongly disagree; $7=$ Strongly agree $)$, and it has been shown to have high internal consistency, good test-retest reliability after 3 weeks, and good convergent and divergent reliability among Western samples (Henderson-King and Henderson-King 2005). Because a number of items were cross-loaded onto more than one factor with Korean samples (Swami et al. 2012b), a total ACSS score obtained by taking the mean of all 15 items, representing the overall acceptance of cosmetic surgery, was a preferable option in the present study in order to measure the extent to which participants showed positive attitudes toward cosmetic surgery. Cronbach's coefficient alphas for the total ACSS were Korea $=.94$ and US $=.95$.

The photographic figure rating scale (Swami and Chamorro-Premuzic 2008). The PFRS consists of 10 grey scale photographic images of real women representing the full range of BMI categories. Participants were asked to complete the scale by rating the figure that most closely matched their own body and the figure that they would most like to possess $(1=$ Figure with the lowest BMI; $10=$ Figure with the highest BMI). A measure of actual-ideal weight discrepancy was computed as the difference between current and ideal ratings. Previous work has shown that the PFRS is cross-culturally valid (Swami, Henderson, et al in press) and has good construct validity and good test-retest reliability after 3 weeks (Swami et al. 2008a).

The body appreciation scale (Avalos et al. 2005). The BAS is a 13-item measure of positive body image of which items are rated on a 5 -point scale $(1=$ Never; $5=$ Always $)$. 
Among Western samples, the BAS has been shown to have good discriminant, construct, and incremental validities (Avalos et al. 2005; Swami et al. 2008b). Cronbach's coefficient alphas for the BAS were Korea $=.90$ and US $=.92$.

The sociocultural attitudes towards appearance questionnaire-3 (SATAQ-3; Thompson et al. 2004). This is a 30-item scale measuring the multi-dimensional impact of sociocultural influences on body image, in which items are rated on a 5-point scale $(1=$ Strongly disagree; $5=$ Strongly agree). Among Western samples, the scale has been shown to consist of four factors measuring the degree to which various types of media are considered an important source of information about being attractive (Information; e.g., 'TV programs are an important source of information about fashion and "being attractive".), feeling pressured by various types of media to strive for cultural ideals of beauty (Pressure; e.g., 'I've felt pressure from TV or magazines to lose weight.'), endorsement and acceptance of media messages touting unrealistic ideals for female beauty and the striving towards these ideals (Internalization-General; e.g., 'I compare my appearance to the appearance of TV and movie stars.), and endorsement and acceptance of an athletic and toned body ideal (Internalization-Athletic; e.g., 'I wish I looked as athletic as sports stars'). Among Western samples, the scale has been shown to be internally reliable and to have good discriminant and convergent validity (Thompson et al. 2004). Cronbach's coefficient alphas for the SATAQ were information: Korea $=.55$, US $=.57$; pressure: Korea $=.92$, US $=.89$; Internalization-General: Korea $=.89$, US $=.89$; InternalizationAthletic: Korea $=.60$, US $=.77$. Given low to moderate levels of reliability for information and internalization-athletic, only pressure and internalization-general were used as sociocultural influences on body image.

\section{Results and discussion}

Since there was a significant difference in participants' body sizes across the two countries and cosmetic surgery has an association with negative body image including body size dissatisfaction, BMI was used as a covariate for a multivariate analysis of covariance (MANCOVA) for the effect of country on attitudes toward celebrities, acceptance of cosmetic surgery, measures of body image, and sociocultural influences on body image. The MANCOVA revealed an overall significance, $F(8,310)=29.72, p<.001$, partial eta ${ }^{2}=.402$. Further examination of analysis of variances revealed significant effects of country on the following measures: The celebrity attitude scale, current figure, ideal figure, current-ideal figure discrepancy on the photographic figure rating scale, the acceptance of cosmetic surgery, and the body appreciation scale. No statistically significant differences were found across the two countries on sociocultural influences (i.e., pressure and internalization of body ideals in the media) on body image. While the South Korean participants had smaller current figures $[M=3.72, S D=1.38$ for Korea; $M=4.15, S D=1.21$ for US, $F(1,317)=6.94, p<.01$, partial eta $\left.{ }^{2}=.021\right]$, they desired to have smaller bodies as their ideals $[M=2.38, S D=.85$ for Korea; $M=3.38, S D=.85$ for US, $F(1,317)=107.73, p=.001$, partial eta $\left.{ }^{2}=.254\right]$, and showed greater currentideal figure discrepancies, $[M=1.34, S D=1.24$ for Korea; $M=.76, S D=1.08$ for US, $F$ $(1,317)=21.72, p<.001$, partial eta $\left.{ }^{2}=.064\right]$. According to the body appreciation scale (BAS), the US participants revealed greater satisfaction toward their bodies $(M=46.24$, $S D=8.19)$ than did the Korean participants $(M=42.04, S D=8.61), F(1,317)=19.76$, 
$p<.001$, partial eta ${ }^{2}=.059$. These results supported Hypothesis 1 , which predicted that the South Korean participants would show greater body dissatisfaction than the US participants.

As further results of the effects of country on attitudes toward celebrities and acceptance of cosmetic surgery, the Korean participants showed greater acceptance toward cosmetic surgery $(M=69.16, S D=18.52)$ than did the US participants $(M=54.56$, $S D=20.41), F(1,317)=41.29, p<.001$, partial eta $^{2}=.116$. This result supported Hypothesis 2: The South Korean participants will show greater acceptance of cosmetic surgery than the US participants. The US participants revealed more positive attitudes toward their favorite celebrities $(M=74.10, S D=19.44)$ than did the Korean participants $(M=65.83, S D=19.46), F(1,317)=15.45, p<.001$, partial eta ${ }^{2}=.047$. This outcome did not support Hypothesis 3: the South Korean participants will show greater celebrity worship than the US participants.

In order to examine which of the relevant factors might explain the participants' acceptance of cosmetic surgery, a multiple regression analysis was run for each country on the acceptance of cosmetic surgery with measures of body satisfaction, attitude toward a favorite celebrity, and sociocultural influences on body image as predictors. The body appreciation scale (BAS) and the participants' current-ideal figure discrepancies were used as measures of body satisfaction, along with the celebrity attitude scale (CAS) and Pressure and Internalization-General for sociocultural influences on body image. BMI was also used as a predictor variable for the participants' acceptance of cosmetic surgery. For the Korean participants, overall predictor variables showed no statistical significance with the acceptance of cosmetic surgery. However, for the US participants, overall statistical significance was obtained with the level of body satisfaction and attitudes toward favorite celebrities being significantly associated with acceptance of cosmetic surgery $\left[F(4,158)=8.25, p<.001, \mathrm{R}^{2}=.168\right]$. The level of body satisfaction measured by the Body Appreciation Scale (BAS) was negatively associated with the acceptance of cosmetic surgery $(\beta=-.287, t=-3.767, p=.001)$, whereas the attitude toward a favorite celebrity, measured by the celebrity attitude scale (CAS), was positively associated with the acceptance of cosmetic surgery $(\beta=.233, t=3.233$, $p=.001$ ) (see Table 2). Thus, these results supported Hypothesis 4-a: The more negative body image participants show the greater likelihood they will engage in cosmetic surgery and Hypothesis 4-b: The more celebrity worship participants show the greater likelihood they will engage in cosmetic surgery in the US participants, but not in the Korean participants.

Similar to findings from a previous study for the relationship between attitudes toward celebrities and acceptance for cosmetic surgery (Swami et al. 2009b), acceptance for cosmetic surgery was related to attitudes toward favorite celebrities in the present study. However, this was evident from the US participants only. Acceptance for cosmetic surgery was also highly related to the degree of body appreciation among the US participants: The less participants appreciate their current bodies, the more likely they are to accept cosmetic surgery. Although participants from both countries did not differ in the degree of which they felt pressured to emulate the cultural standards of ideal beauty, the Korean participants showed a greater acceptance for cosmetic surgery than the US participants. This suggests that Korean women are more willing to undergo cosmetic 
Table 2 Multiple regression with acceptance of cosmetic surgery scale (ACSS) as the dependent variable

\begin{tabular}{|c|c|c|c|c|c|c|}
\hline \multirow{2}{*}{$\begin{array}{l}\text { Country } \\
\text { Measures }\end{array}$} & \multicolumn{3}{|l|}{ US } & \multicolumn{3}{|l|}{ Korea } \\
\hline & Standardized $\beta$ & $t$ & $p$ & Standardized $\beta$ & $t$ & $p$ \\
\hline BMI & -.100 & -1.138 & .257 & -.017 & -.211 & .833 \\
\hline Body appreciation scale (BAS) & -.287 & -3.767 & .000 & -.186 & -1.979 & .049 \\
\hline $\begin{array}{l}\text { Current-ideal figure discrepancy (photo- } \\
\text { graphic figure rating scale) }\end{array}$ & .112 & 1.228 & .221 & -.129 & -1.354 & .177 \\
\hline Celebrity attitude scale (CAS) & .233 & 3.233 & .001 & .088 & 1.182 & .239 \\
\hline
\end{tabular}

procedures to improve their physical appearance, and/or perhaps there is less social stigma or greater acceptance about cosmetic surgery in Korea than in the US contemporary South Korean society is highly advanced with technology, as South Korea is ranked the third in the world's most technologically advanced countries (Most technologically advanced countries 2015). Unlike the traditional Korean society that limited women's roles to the household with little power beyond domestic activities (Jung 2003), a large majority of South Korean women attend college, which is described as the highest rate in the world (Lee and Caryl 2005). A greater level of acceptance for cosmetic surgery among the Korean sample than the US sample also suggests a greater emphasis on physical attractiveness in Korea, and Koreans may use physical attractiveness as a means to obtain opportunities for jobs and marriage, as well as self-acceptance (Choe 2011). Great emphasis on appearance and desire to achieve success, coupled with advanced medical technology, are suggested to stimulate cosmetic surgery practices in South Korea.

Although the Korean participants showed greater dissatisfaction with their figures (i.e., body shapes and sizes) than the US participants, their level of body dissatisfaction was not a factor influencing the acceptance of cosmetic surgery, unlike the result for the US participants. This is interesting given that previous studies conducted in Western samples showed positive relationships between negative body image and the consideration of cosmetic surgery. To some extent this can be attributed to the uniqueness of East Asian beauty standards of which women's physical attractiveness is greatly focused on facial features rather than bodily characteristics such as shape and weight (Lee 1999). In fact, for many years the most popular cosmetic surgery procedures in Korea have been eyelid surgery and rhinoplasty which target changing facial characteristics (Kim 2003). However, according to recent statistics, the two most commonly performed surgical procedures in Korea were lipoplasty, also known as liposuction (removing body fat), along with eyelid surgery (ISAPS Global Statistics 2014). Recent statistics also indicate an increasing demand for breast augmentation in South Korea (ISAPS Global Statistics). These types of surgical procedures coincide with Western body ideals of the 'curvaceously thin' woman. Thus, it is feasible to foresee that Korean women's beauty standards emphasize not only facial attractiveness but also obtainment of idealized figures. 


\section{Conclusions}

A positive association between attitudes toward celebrities and the acceptance of cosmetic surgery among the US participants implies that compared to South Korea, celebrities in the US might have a stronger effect as a reference group for ideal appearance standards and play a greater role for one's likelihood of having cosmetic surgery. Also, messages/articles regarding beauty products and related programs (e.g., diet programs) that are pervasive in the mass media, particularly those endorsed by favorite celebrities, might have a stronger effect in the US than in South Korea. This was also supported by greater positive attitudes toward favorite celebrities in the US compared to Korea. While celebrities have helped to drive the trend of remedying any physical imperfections (Choe 2011), with a large number of practices in South Korea motivations to engage in cosmetic surgery may be attributed to beyond celebrity influences. In fact, competitiveness for success and jobs is suggested as a driving factor for cosmetic surgery boom in South Korea (CBS News 2015). Overall, findings of this study suggest that the cultural environment influences factors affecting the likelihood of having cosmetic surgery.

As for almost all cross-cultural research, our study is limited by samples of college students that are not representative of their countries as a whole. College women are generally a privileged group that is younger, wealthier, and more likely to be exposed to Western body ideals than the general population. Consequently, the results of this study should be generalized with appropriate caution. In addition, we used measures that were developed in Western cultures and translated from their original English. Our translations were carefully done using the back-translation technique (Breslin 1970) for cross-cultural research, and the reliability of the translated measures was very similar to the reliability of the original English version. However, translations are always potentially problematic and should be interpreted with appropriate care (King 1993). There are other cultural factors that might influence the likelihood of having cosmetic surgery, such as the price of cosmetic surgery procedures and reputation of cosmetic surgeons. Also, cultural differences in other individual perceptions about cosmetic surgery (e.g., being unethical, fear for unknown outcomes) can influence individual responses to the likelihood of having cosmetic surgery.

Further research on this topic can examine gender differences along with country differences since an increasing number of men are undergoing cosmetic surgery procedures as a way to improve their appearance. In fact, According to the American Society for Aesthetic Plastic Surgery (2015), the number of men who underwent aesthetic cosmetic procedures is up $43 \%$ over a five-year period for both surgical and non-surgical procedures. Just as women are not immune to body image dissatisfaction, more men seem to be interested in cosmetic procedures to maintain their youthful looks.

Attitudes toward cosmetic surgery can be examined by employing one-on-one or focus group interviews to delve into individuals' motivations for having cosmetic surgery and the effects that their culture has on them. Further research can also examine consumers' motivations to engage in specific cosmetic surgery procedures (e.g., breast or buttock augmentation) across countries, which can provide richer psychological or socio-cultural factors that would influence cosmetic surgery.

Authors' contributions

JJ designed and carried out research and drafted the manuscript. CSH assisted with data collection from South Korea. Both authors read and approved the final manuscript. 


\section{Author details \\ ${ }^{1}$ University of Delaware, Newark, USA. ${ }^{2}$ Kyung Hee University, Seoul, South Korea.}

\section{Competing interests}

The authors declare that they have no competing interests.

Received: 18 February 2016 Accepted: 15 July 2016

Published online: 28 August 2016

\section{References}

Abraham, A., \& Zuckerman, D. (2011). Adolescents, celebrity worship, and cosmetic surgery. Journal of Adolescent Health, $49,453-454$.

American society for aesthetic plastic surgery. (2015). The American society for aesthetic plastic surgery reports americans spent largest amount on cosmetic surgery since the great recession of 2008. http://www.surgery.org/media/ statistics.

American society of plastic surgeons (2014). Plastic surgery procedures continue steady growth in US http://www. plasticsurgery.org/news/plastic-surgery-procedures-continue-steady-growth-in-us.html.

Avalos, L., Tylka, T. L., \&Wood-Barcalow, N. (2005). The body appreciation scale: development and psychometric evaluation. Body Image, 2, 285-297.

Bell, D. A., \& Chaibong, H. (2003). The contemporary relevance of Confucianism. In D. A. Bell \& H. Chaibong (Eds.), Confucianism for the modern world (pp. 1-28). Cambridge: Cambridge University Press.

Breslin, R. W. (1970). Back-translation for cross-cultural research. Cross-Cultural Psychology, 1, 185-216.

Brown, A., Furnham, A., Glanville, L., \& Swami, V. (2007). Factors that affect the likelihood of undergoing cosmetic surgery. Aesthetic Surgery Journal, 27, 501-508.

CBS News. (2015). Competitive culture triggers plastic surgery boom in S. Korea. CBSN. http://www.cbsnews.com/news/ plastic-surgery-south-korea-obsession-competitive-culture/.

Choe, S-H. (2011). In South Korea, plastic surgery comes out of the closet. The New York Times. http://www.nytimes. com/2011/11/04/world/asia/in-south-korea-plastic-surgery-comes-out-of-the-closet.html?pagewanted=all.

Cook, S. A., Rosser, R., \& Salmon, P. (2006). Is cosmetic surgery an effective psychotherapeutic intervention? A systematic review of the evidence. Journal of Plastic and Reconstructive Aesthetic Surgery, 59, 1133-1151.

Crerand, C. E., Franklin, M. E., \& Sarwer, D. B. (2006). Body dysmorphic disorder and cosmetic surgery. Plastic and Reconstructive Surgery, 118, 167-180.

Delinksy, S. S. (2005). Cosmetic surgery: a common and accepted form of self-improvement? Journal of Applied Social Psychology, 35, 2012-2028.

Dent, M. (2016, March 9). Plastic surgery reaches a new record: The top 5 nip-and-tucks in 2015. The Fiscal Times. Retrieved from http://www.thefiscaltimes.com/2016/03/09/Plastic-Surgery-Reaches-New-Record-Top-5 -Nip-and-Tucks-2015

Durkin, S. J., Paxton, S. J., \& Sorbello, M. (2007). An integrative model of the impact of exposure to idealized female images on adolescent girls' body satisfaction. Journal of Applied Social Psychology, 37, 1092-1117.

Edmonds, A. (2007). 'The poor have the right to be beautiful': cosmetic surgery in neoliberal Brazil. Journal of the Royal Anthropological Institute, 13, 363-381.

Forbes, G. B., \& Jung, J. (2008). Measures based on sociocultural theory and feminist theory as predictors of multidimensional measures of body dissatisfaction among Korean and US college women. Journal of Social and Clinical Psychology, 27, 63-92.

Frederick, D. A., Lever, J., \& Peplau, L. A. (2007). Interest in cosmetic surgery and body image: views of men and women across the lifespan. Plastic and Reconstructive Surgery, 120, 1407-1415.

Giles, D. C. (2002). Parasocial interaction: a review of the literature and a model for future research. Media Psychology, 4, 279-302.

Giles, D. C., \& Maltby, J. (2004). The role of media figures in adolescent development: relations between autonomy, attachment, and interest in celebrities. Personality and Individual Differences, 36, 813-822.

Grabe, S., Ward, L. M., \& Hyde, J. S. (2008). The role of the media in body image concerns among women: a meta-analysis of experimental and correlational studies. Psychological Bulletin, 134, 460-476.

Greenwood, D. (2009). Idealized TV friends and young women's body concerns. Body Image, 6, 97-104.

Harrison, K. (2003). Television viewers' ideal body proportion: the case of the curvaceously thin woman. Sex Roles, 48, 255-264.

Henderson-King, D., \& Henderson-King, E. (2005). Acceptance of cosmetic surgery: scale development and validation. Body Image, 2, 137-149.

ISAPS global statistics. (2014). ISAPS international survey on aesthetic/cosmetic procedures performed in 2014. International Society of Aesthetic Plastic Surgery. http://www.isaps.org/press-center/isaps-global-statistics.

Jung, K. (2003). Practicing feminism in South Korea: the issue of sexual violence and the women's movement. Hecate, 29, 261-284.

Jung, J., \& Forbes, G. (2006). Multidimensional assessment of body dissatisfaction and disordered eating in Korean and US college women: a comparative study. Sex Roles, 55, 39-50.

Jung, J., Forbes, G. B., \& Lee, Y-J. (2009). Body dissatisfaction and disordered eating among early adolescents from South Korea and the United States. Sex Roles: A Journal of Research, 61, 42-54. doi:10.1007/s11199-009-9609-5.

Kim, T. (2003). Neo-confucian body techniques in Korea's consumer society. Body and Society, 9, 97-113.

Kim, O., \& Kim, K. (2003). Comparisons of body mass index, perception of body weight, body shape satisfaction, and selfesteem among Korean adolescents. Perceptual and Motor Skills, 97, 1339-1346.

King, M. B. (1993). Cultural aspects of eating disorders. International Review of Psychiatry, 5, 205-216. 
Lee, S. (1999). Fat, fatigue and the feminine: the changing cultural experience of women in Hong Kong. Culture, Medicine and Psychiatry, 23, 51-73.

Lee, S. J. (2011). The Korean wave: the Seoul of Asia. The Elon Journal of Undergraduate Research in Communications, 2, 85-93.

Lee, B.J., Caryl, C. (2005, May 16). Rising to the top: after enduring decades of discrimination, South Korean women are surging into positions of power and influence. Newsweek International, p. 32.

Maltby, J., \& Day, L. (2011). Celebrity worship and incidence of elective cosmetic surgery: evidence of a link among young adults. Journal of Adolescent Health, 49, 483-489.

McCurdy, J. A., \& Lam, S. M. (2005). Cosmetic surgery of the Asian face (2nd ed.). New York: Thieme Medical Publishers.

McCutcheon, L. E., Lange, R., \& Houran, J. (2002). Conceptualization and measurement of celebrity worship. British Journal of Psychology, 93, 67-87.

Most technologically advanced countries. (2015). http://richestlifestyle.com.

Rye, H. R., Lyle, R. M., \& McCabe, G. P. (2003). Factors associated with weight concerns and unhealthy eating patterns among young Korean females. Eating Disorders, 11, 129-141.

Sarwer, D. B., Cash, T. F., Magee, L., Williams, E. F., et al. (2005). Female college students and cosmetic surgery: an investigation of experiences, attitudes, and body image. Plastic and Reconstructive Surgery, 115, 931-938.

Sarwer, D. B., Crerand, C. E., \& Gibbons, L. M. (2007). Cosmetic surgery to enhance body shape and muscularity. In J. K. Thompson \& G. Cafri (Eds.), The muscular ideal: Psychological, social, and medical perspectives (pp. 183-198). Washington, DC: American Psychological Association.

Sarwer, D. B., \& Magee, L. (2006). Physical appearance and society. In D. B. Sarwer, T. Pruzinsky, T. F. Cash, R. M. Goldwyn, J. A. Persing, \& L. A. Whitaker (Eds.), Psychology of reconstructive and cosmetic plastic surgery: clinical, empirical, and ethical perspectives (pp. 23-36). Philadelphia: Lippincott, Williams, \& Wilkins.

Shim, D. (2006). Hybridity and the rise of Korean popular culture in Asia. Media, Culture and Society, 28, 25-44.

Shin, D. C., \& Rutkowski, C. P. (2003). Subjective quality of Korean life in 1981 and 2001. Social Indicators Research, 62, 509-534.

Shorter, L., Brown, S. L., Quinton, S. J., \& Hinton, L. (2008). Relationships between body-shape discrepancies with favored celebrities and disordered eating in young women. Journal of Applied Social Psychology, 38, 1364-1377.

Swami, V., Arteche, A., Chamorro-Premuzic, T., Furnham, A., Stieger, S., Haubner, T., et al. (2008a). Looking good: factors affecting the likelihood of having cosmetic surgery. European Journal of Plastic Surgery, 30, 211-218.

Swami, V., \& Chamorro-Premuzic, T. (2008). Factor structure of the body appreciation scale among Malaysian women. Body Image, 5, 409-413.

Swami, V., Chamorro-Premuzic, T., Bridges, S., \& Furnham, A. (2009a). Acceptance of cosmetic surgery: personality and individual difference predictors. Body Image, 6, 7-13.

Swami, V., Campana, A. N., \& Coles, R. (2012a). Acceptance of cosmetic surgery among British female university students. European Psychologist, 17, 55-62. doi:10.1027/1016-9040/a000049.

Swami, V., Hwang, C. S., \& Jung, J. (2012b). Factor structure and correlates of the acceptance of cosmetic surgery scale among South Korean university students. Aesthetic Surgery Journal, 32, 220-229. doi:10.1177/1090820X11431577.

Swami, V., Stieger, S., Haubner, T., \& Voracek, M. (2008b). Translation and validation of the German body appreciation scale. Body Image, 5, 122-127.

Swami, V., Taylor, R., \& Carvalho, C. (2009b). Acceptance of cosmetic surgery and celebrity worship: evidence of associations among female undergraduates. Personality and Individual Differences, 47, 869-872.

Sypeck, M., Gray, J., \& Ahrens, A. (2004). No longer just a pretty face: fashion magazines' depictions of ideal female beauty from 1959 to 1999. International Journal of Eating Disorders, 36, 342-347.

Thompson, J. K., van den Berg, P., Roehrig, M., Guarda, A. S., \& Heinberg, L. J. (2004). The sociocultural attitudes toward appearance questionnaire (SATAQ-3): development and validation. International Journal of Eating Disorders, 35, 293-304.

White, A. (2005, March 11). Man-made faces front Korean campaigns. Media Asia, p. 20.

\section{Submit your manuscript to a SpringerOpen ${ }^{\circ}$ journal and benefit from:}

- Convenient online submission

Rigorous peer review

- Immediate publication on acceptance

- Open access: articles freely available online

- High visibility within the field

- Retaining the copyright to your article

Submit your next manuscript at $>$ springeropen.com 